\title{
Gun Shot Residue Analysis and Distinguishing the formation of GSR from Environmental Particles
}

\author{
Ivan Sarvas*, Hilton Kobus**, Len Green*** Paul Kotula**** and Richard Wuhrer***** \\ * Forensic Science South Australia (FSSA) Adelaide. \\ ** School of Chemistry, Physics and Earth Science, Flinders University \\ *** Adelaide Microscopy, The University of Adelaide, South Australia \\ ****Sandia National Laboratories, Albuquerque, New Mexico, USA. \\ ***** Microstructural Analysis Unit, University of Technology, Sydney.
}

Police investigations and the criminal judicial system including civil litigation rely on Forensic Science to assist them to answer or shed some light on:

1. What happened?

2. Who did what?

3. Where, when and how?

The detection and identification of gun shot residue (GSR) particles on suspects, victims and objects provide information to assist the police in their investigations and these results are ultimately tested by the judicial system. GSR particles are analysed in laboratories by scanning electron microscopy (SEM) with an energy dispersive spectrometer (EDS) and more recently using silicon drift detectors (SDD's), coupled to an automatic particle search and analysis system. The SEM/EDS/SDD analysis provides non-destructive elemental analysis and morphological characterization of particles. The use of the new generation of SDD's allows for faster analysis and particle mapping (4 to 10 times faster).

GSR particles typically exhibit a molten and condensed surface appearance of some complexity and may contain the elements lead, antimony and barium (Figures 1 and 2). These elements are derived from inorganic compounds of the primer containing lead styphnate, barium nitrate and antimony sulphide. GSR particles can also contain a mixture of elements derived from the bullet, cartridge case and contaminants from previous firings.

In recent years, studies have indicated that characteristic GSR particles and GSR particles produced by non-toxic primers may be indistinguishable from environmental particles. It has been reported that some particles produced by the wear and elevated temperatures of brake linings are similar or indistinguishable from primer residue. Studies have also implicated pyrotechnics and fireworks as a possible source.

GSR particles are formed in a supersaturated environment of high temperature and pressure, and brake lining particles are produced by friction hotspots due to heavy braking. The temperature of brake linings in normal traffic situations is about $200-300^{\circ} \mathrm{C}$. In intense braking, test temperatures are elevated well above $900^{\circ} \mathrm{C}$. Because GSR particles are formed from rapid cooling from extreme temperatures and pressures, it is expected that these particles contain condensates not only on their surfaces but also within their interiors. The processes resulting in the formation of GSR particles and brake linings are quite different and produce particles of different morphological and compositional structures that will allow for their discrimination.

Cross-sectioning of particles through use of focused ion beam milling (FIB) and analysis by electron beam allows the analyst to reveal the interior morphology and elemental profile to provide information 
about the processes of formation. By extracting both elemental and morphological information form the core and interior, the analyst might be able to differentiate environmental sources and GSR particles.

This paper will present results from SEM and FIB investigations of GSR particles and other environmental particles.

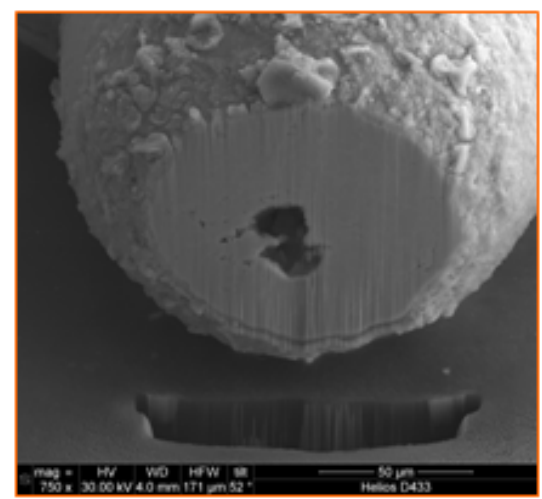

Weld Slag

a.

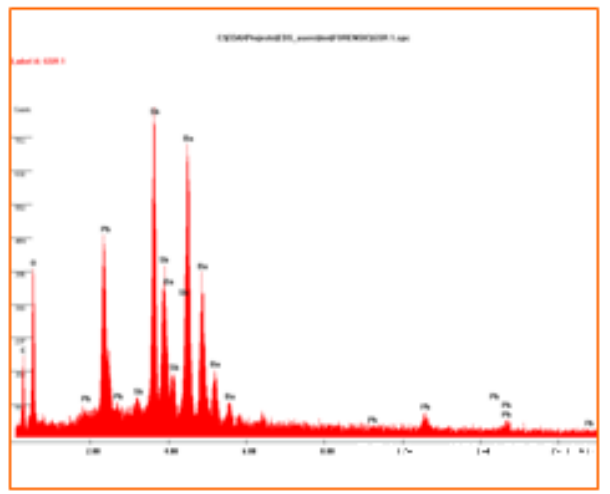

d.

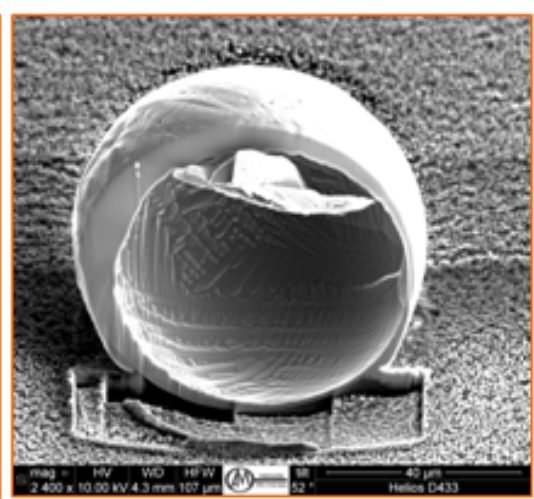

Pyrotechnic-sparkler

b.

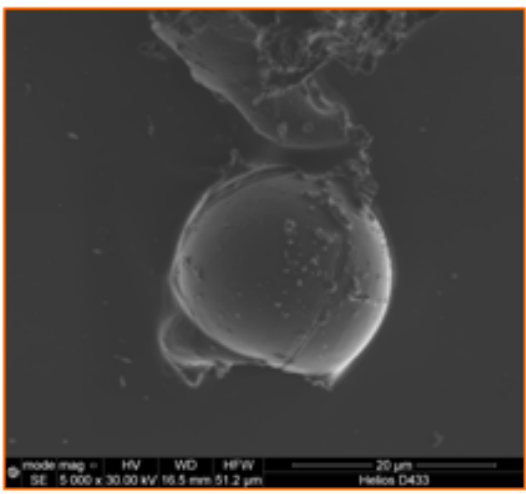

e.

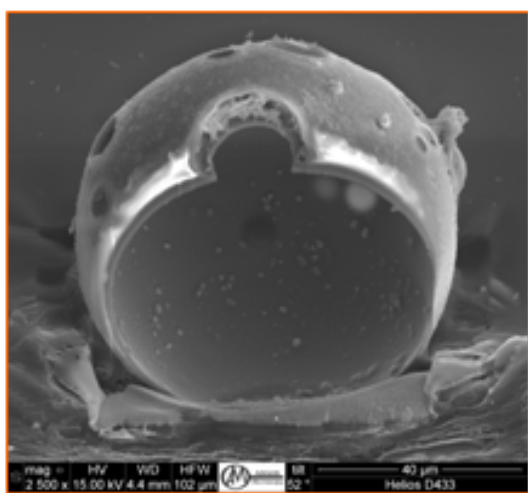

Gun Shot Residue

c.

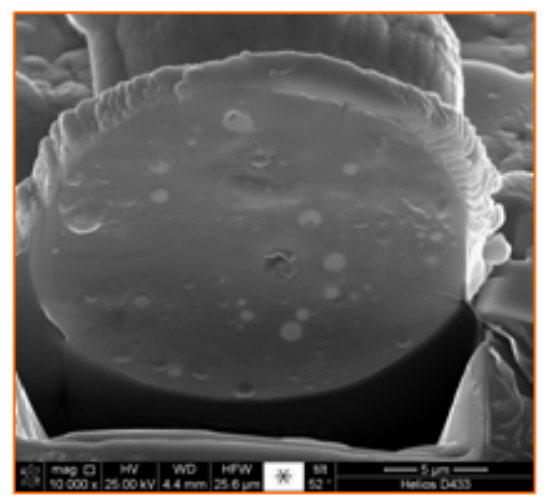

f.

Fig. 1: FIB, SEM and EDS analysis results indicating internal morphology of three different types of particles. a) Weld slag particle, b) pyrotechnic sparkler particle, c) GSR particle, d) EDS spectrum of GSR particle, e) SEI image of GSR particle and f) FIB section of GSR particle.

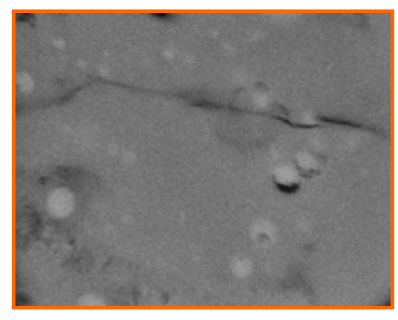

a.

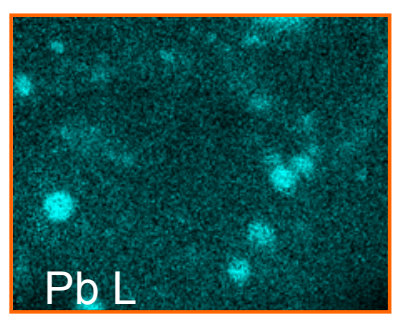

b.

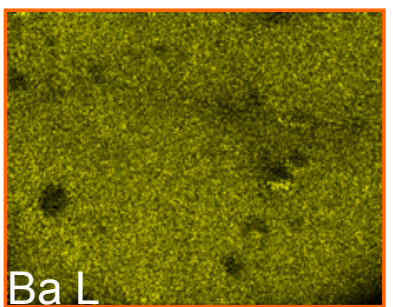

c.

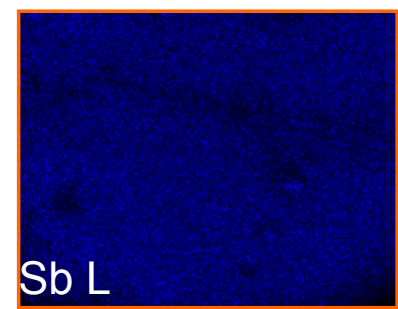

d.

Fig. 2: Analysis of GSR particle a) SEI Image of GSR particle, b) Pb L line x-ray map, c) Ba L line xray map and d) Sb L line X-ray map. 